Sports Training

\title{
The effects of active vs. passive recovery on riding performance during a simulated motocross race
}

\author{
Aleix Olle-Casanovas ${ }^{1}$ (D) , Juha P. Ahtiainen ${ }^{1}$ (iD \\ ${ }^{1}$ University of Jyväskylä, Neuromuscular Research Center, Faculty of Sport and Health Sciences, \\ Jyväskylä, Finland.
}

Associate Editor: Eduardo Lusa Cadore, Universidade Federal do Rio Grande do Sul, UFRGS, Porto Alegre, RS, Brasil.

\begin{abstract}
Aims: Motocross consists of two races of 30 min with a break in between. Recovery between races is paramount to performance, this study aimed to compare the effects of active and passive recovery between motocross races on riding performance. Methods: Thirteen elite and non-elite racers performed two races of $30 \mathrm{~min}$ with $1 \mathrm{~h}$ break, twice with two-week interval. Between the races active ( 20 min ergometer cycling at $60 \%$ of HRmax) or passive recovery (no activity) were executed in a randomized order. Lap times, heart rate during riding, blood lactate, reaction time, handgrip strength, upper back pull strength, counter movement jump height, and medicine ball throw distance were measured before and after both races. Serum creatine kinase enzyme activity and strength tests were assessed also $24 \mathrm{~h}$ after the races. Results: No statistically significant differences were observed in any variables between protocols. A significant drop in handgrip strength was found post-race values $(\mathrm{p}<0.001)$. Post-racing creatine kinase values were above resting levels. Conclusion: These results do not support the use of active recovery in between motocross races for restoration of neuromuscular or riding performance. Motocross seems to cause some exercise-induced muscle damage; thus, some rest is required to recover homeostasis.
\end{abstract}

Keywords: handgrip strength, muscle damage.

\section{Introduction}

In motocross racing the riders have to manoeuvre a heavy and powerful motorcycle in order to go as fast as possible on a dirt track. Most of the muscular actions are isometric and eccentric, when the rider accelerates and brakes while absorbing the shocks created by the uneven and rough terrain and the jumps ${ }^{4,18}$. Previous research findings show that motocross riding requires great levels of strength and endurance $e^{3,19,18}$.

It seems that strength is impaired after a motocross bout, and the forearm muscles have showed maximal EMG activity during racing ${ }^{3,19,18}$. Moreover, heart rate (HR) and breathing frequency are near $100 \%$ for the majority of the time, racers oxygen consumption $\left(\mathrm{VO}_{2}\right)$ stays around $70 \%$ of $\mathrm{VO}_{2}$ max, and blood lactate concentrations are close to the anaerobic threshold ${ }^{4,3,8,18,19}$. Ascensão et al. ${ }^{4}$ found a considerable increase in catecholamine plasma levels. This increase seems to be a contributor to muscular force expression, high HR values and large glucose production ${ }^{15,20,39}$. Isometric muscle actions during motocross riding can play a significant role in increasing blood pressure, heart rate and ventilation ${ }^{27,30}$.

Active recovery shows faster lactate removal ${ }^{10}$, but lactate removal does not appear to be a valid indicator of recovery quality ${ }^{6}$. Active recovery in between ski runs, swimming bouts and high intensity interval training seems to be superior to maintain performance and work capacity $^{1,11,37}$. Greater $\mathrm{O}_{2}$ availability may be one of the causes why active recovery could increase performance in the following exercise bout ${ }^{28}$. Previous research show no or little benefit when active recovery is performed between football training sessions, cross country skiing bouts, basketball sprint training and high intensity walking ${ }^{16,21,26,31,33}$. Passive recovery may increase performance or training induced adaptations during football matches, repeated sprint training, high intensity cycling bouts and triathlon ${ }^{2,29,35,36}$. It seems that active recovery might slow glycogen resynthesis ${ }^{12}$. However, the effects of active and passive recovery between motocross bouts are unknown.

Exercise induced muscle damage (EIMD) affects the muscles by altering the organization of the myofibers, and resulting in decreases in strength and range of motion, soreness and swelling, and efflux of myocellular proteins $^{24}$. This alteration normally happens when subjects perform unaccustomed exercise and this effect is accentuated with eccentric muscle actions ${ }^{24}$. Muscle actions during motocross racing are both isometric and eccentric indicating possible EIMD after racing. EIMD can impair neuromuscular performance for a few days ${ }^{5}$. 
The purpose of this study was to examine the acute effects of active and passive recovery between motocross races on neuromuscular and riding performance. EIMD was also assessed a day after the experimental motocross races. We hypothesize that active recovery protocol will be superior to passive recovery on motocross riding and neuromuscular performance outcomes. We also hypothesize that the degree of muscle damage will be lower in the active recovery group

\section{Methods}

\section{Experimental approach to the problem}

The goal of this cross-sectional study with repeated measures design was to examine the role of active and passive recovery in between motocross races, and its effect on riding performance, strength, power, reaction time and muscle damage. National level racers and recreational riders simulated a motocross race with 2 riding bouts of $30 \mathrm{~min}$ separated by $1 \mathrm{~h}$. Participants were assessed with different performance tests before and after riding, such as blood lactate, grip strength, upper body pull strength, counter movement jump, reaction time and seated medicine ball throw. Heart rate and lap times were recorded while riding. The riders were randomly assigned either to active or passive recovery group during the $1 \mathrm{~h}$ break. The same tests were performed during the second riding bout. This protocol was repeated 15 days later, with the group that performed active recovery being assigned to the passive recovery protocol and vice versa. One day after both simulated motocross races, participants repeated the strength and power tests done at the track and serum creatine kinase enzyme samples were taken from the fingertip.
Subjects

Eleven $(\mathrm{n}=11) 2$ national level racers and 9 amateur male racers volunteered in this study (average age; $33 \pm$ 10 years, height; $179 \pm 7 \mathrm{~cm}$, body mass; $80.8 \pm 10.0 \mathrm{~kg}$, and body mass index; $25 \pm 2$ ). All participants had at least 5 years of motorcycle riding and racing experience. Participants were informed about the different protocols and measurements used in this study, as well as the possible risks. Written informed consent was obtained from all subjects. The study was conducted according to the Declaration of Helsinki, and ethical statement for the study procedures were granted by the Ethical Committee at the University of Jyväskylä, Finland (523/1999).

\section{Procedures}

Participants performed two simulated motocross races $(30 \mathrm{~min})$ emulating race pace, with $1 \mathrm{~h}$ break in between. The active and passive recovery protocols were used in a randomized order during the break. Active recovery consisted of $20 \mathrm{~min}$ of ergometer cycling at $60 \%$ of HRmax estimated by participant's age ${ }^{32}$ performed immediately after the first simulated race, and $40 \mathrm{~min}$ of resting. HR was monitored throughout the active recovery protocol. When subjects performed the passive recovery protocol, they remained seated without performing any type of physical activity. Active and passive recovery protocols and testing were separated by 2 weeks in order to avoid accumulation of fatigue. The simulated motocross races were performed in the same race track with similar ambient conditions $\left( \pm 5^{\circ} \mathrm{C}\right)$.

Performance tests were done before and after the first and second motocross races (Figure 1). After the first post-test took place, racers executed the assigned recovery protocol.

Simulated Race day

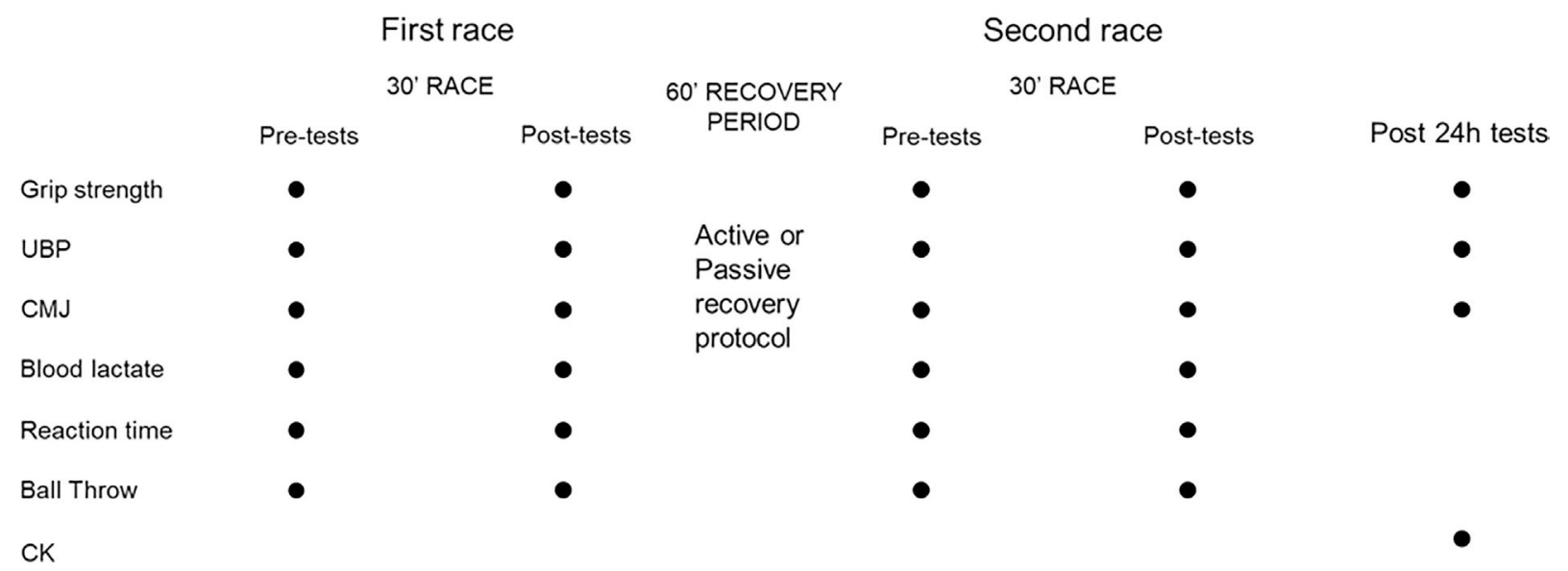

Figure 1 - Testing schedule. Lap times were recorded during the races and fluid intake was controlled by standardized sport drink during the recovery period. The same program was repeated at the same racetrack after 2 weeks. UBP $=$ Upper body pull; CMJ = Counter movement jump; CK $=\mathrm{Creatine}$ Kinase enzyme activity. 
All the subjects consumed the same amount of commercial sports drink during the break containing $60 \mathrm{~g} / \mathrm{L}$ carbohydrates and $50 \mathrm{mmol} / \mathrm{L} \mathrm{Na}^{+22,25}$.

\section{Measurements and analysis}

Track measurements

Lap times were recorded using infrared sensors placed in the finish line of the motocross track and transponders placed on every participant's motorcycle (J2Chrono Timekeeping Envoirment, Enymind Oy, Finland). Heart rate (HR) was monitored continuously during the races and active recovery protocol for all subjects using wireless HR monitors (Polar V800, Oulu, Finland) with a sampling frequency of $1 \mathrm{~Hz}$.

\section{Performance measurements}

All performance measurements were taken before and immediately after each race and their order was: blood lactate, grip strength, upper body pull strength, counter movement jump, reaction time test and seated medicine ball throw test. One research assistant was allocated to each of the measurements. All tests were performed with subjects wearing motorcycle gear and helmet to minimise the time between the end of race and the end of testing.

Maximal isometric strength was assessed using a handgrip test and an upper body pull strength test (UBP). Force data $(\mathrm{N})$ was collected using linear force transducers adapted into two custom-built devices (Department of Biology of Physical Activity of the Jyväskylä University, Finland). Subjects were asked to contract as forceful and as fast as possible for 3- $4 \mathrm{~s}$. The grip strength test was performed with the right hand in a seated position with the shoulder at $0^{\circ}$ of vertical adduction and the elbow at $90^{\circ}$ of flexion. For the UBP, subjects were instructed to pull from a barbell attached to the linear force transducer in a seated position with the knees bent approximately at $45^{\circ}$.

Upper body power was assessed using the distance travelled $(\mathrm{cm})$ by a $5 \mathrm{~kg}$ ball during a seated medicine ball

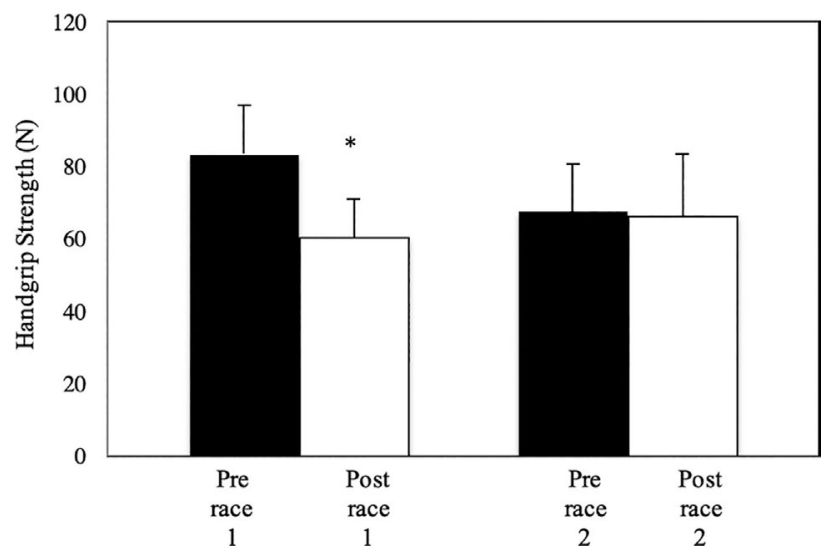

Figure 2 - Pre and Post Race 1 maximal handgrip strength (mean \pm SD). $*$ = statistically significant difference from Pre Race 1 . throw test. Subjects were asked to perform an overhead throw while seated with the back supported against a wall and hips flexed at approximately $90^{\circ}$ degrees. Lower body power was assessed using a countermovement jump (CMJ) height. Flight time ( $\mathrm{t}$ ) was collected using an infrared custom-built device (Department of Biology of Physical Activity of the University of Jyväskylä, Finland). Jump height (h) was then estimated using the following Eq. (1):

$$
h=\frac{1}{2} \cdot g \cdot \frac{t}{2} \cdot 2
$$

where $g=9.81 \mathrm{~m} \cdot \mathrm{s}^{-2}$. Subjects were instructed to jump as high as possible starting from a standing position with their hands on the hips followed by a downwards movement flexing the knees and hips then immediately extending the knees and hips to jump vertically.

Reaction time was assessed using a visual reaction time test using a custom-built device (Department of Biology of Physical Activity of the Jyväskylä University, Finland). While standing upright, subjects were instructed to react to visual stimuli and press a button when the light turned on. The visual stimuli were programmed to appear after a research assistant pressed a button outside the subject's field of vision. Each test consisted of 10 stimuli. Time elapse (s) between the visual stimuli and the moment the subject pressed the button were recorded as a measure of reaction time. Prior to the beginning of the protocol, all the subjects familiarised with the reaction time test for $5 \mathrm{~min}$ to minimise learning effects. The results obtained during the familiarization were not recorded. The average of the 5 quickest times of every participant in every test were used for later analysis.

Fingertip blood lactate concentrations were measured with a portable blood lactate monitor (Lactate Scout +, EKF, Cardiff, England, CV 3,5\%).

Muscle damage was assessed using serum creatine kinase enzyme (CK). Samples were taken from the fingertip $24 \mathrm{~h}$ after the race and stored at $4{ }^{\circ} \mathrm{C}$. CK was analysed less than $24 \mathrm{~h}$ after data collection using a standardised automatic procedure (REF) (Konelab $20 \mathrm{XTi}$, Thermo Fisher Scientific, Vantaa, Finland, CV 1,7\%). Furthermore, CMJ, UBP, and handgrip strength tests were also measured one day after the simulated races.

All data was converted to a .csv format and analysed using Microsoft Excel (Excell 2011, Microsoft, USA).

\section{Statistical analyses}

Data normality was confirmed using a Shapiro-Wilk test. Data are presented as mean and standard deviation (SD). A two-way repeated measures analysis of variance was performed to investigate the effects of time (Pre race 1, Post race 1, Pre race 2, or Post race 2) and recovery protocol (active or passive), on the different measurements. If an effect of time or intervention was observed, Post-hoc tests with a Bonferroni correction were per- 
formed to identify the origin of the differences. Serum creatine kinase concentration levels at $24 \mathrm{~h}$ follow-up were compared between the active and passive recovery protocols using paired t-tests. All statistical analysis was performed using RStudio(v1.1). Significance was set at $\mathrm{p}<0.05$.

\section{Results}

No significant differences between active and passive recovery protocols were found when comparing the results of the different tests. Absolute results and changes are shown in Table 1 and 2.

\section{Lap time}

No significant interactions between time and recovery protocol were observed for lap times (Tables 1-2).

\section{Force and power production}

No significant interactions between time and recovery protocol were observed for $\mathrm{CMJ}$ height $(\mathrm{F}(1.51,12.1)$ $=1.076, \mathrm{p}=0.352)$, UBP isometric strength $(\mathrm{F}(4,32)=$ $0.557, \mathrm{p}=0.695)$, handgrip isometric strength $(\mathrm{F}(4,32)=$ $1.004, \mathrm{p}=0.420)$, or medicine ball throwing distance $(\mathrm{F}(3,24)=0.0627, \mathrm{p}=0.605)$. (Table 1-2). However, we observed significant effects of time for handgrip strength $(\mathrm{F}(3,24)=18.083, \mathrm{p}<0.001)$. Post-hoc comparison revealed that handgrip strength was decreased from Pre to Post race $1(\mathrm{p}<0.05)$ and did not recover to baseline values at Pre race 2 for either the passive or active recovery protocols (Table 1-2). Additionally no significant differences in grip strength were observed between Post race 1 and Post race 2. At $24 \mathrm{~h}$ follow-up, handgrip strength was not statistically significant from Pre race 1 for both the recovery protocols $(\mathrm{p}>0.05)$.

\section{Blood lactate concentrations and heart rate}

No significant interactions between time and recovery protocol were observed for either blood lactate concentration $(\mathrm{F}(3,24)=1.57, \mathrm{p}=0.23)$ or mean HR during the race $(F(1,8)=2.162, p=0.180)$. However, a main effect of time was observed for both blood lactate concentrations $(\mathrm{F}(2.01,16.12)=16.656, \mathrm{p}<0.001)$. Post-hoc comparisons showed a significant increase in blood lactate concentrate after the first race in both recovery protocols $(\mathrm{p}<0.05)$ (Figure 3) and no significant differences between Pre race 1 and Pre race 2 for either passive or active recovery protocols (Table 1-2). Post race 2, a significant increase in blood lactate concentration was only observed for the active recovery protocol $(p=0.043)$.

\section{Reaction time}

No significant interactions between time and recovery protocol were observed for RT $(\mathrm{F}(3,24)=0.833$, $\mathrm{p}=0.489)$, however a main effect of time was observed $(\mathrm{F}(2.02,16.17)=17.295, \mathrm{p}<0.001)$. Post-hoc analysis revealed a significant reduction in RT at Post race 2 compared to Pre race 1 and a Post race 1 for both passive and active recovery groups (Tables 1 and 2).

\section{Creatine kinase and strength tests 24 h post-race}

The strength and power tests (grip strength, $\mathrm{CMJ}$ and UBP) did not indicate any significant differences when comparing active or passive recovery. Serum creatine kinase enzyme results $24 \mathrm{~h}$ after the simulated race (mean $\pm \mathrm{SD}$ ), did not show a significant difference between groups. Passive recovery group $(513 \pm 540 \mu \mathrm{L})$ and active recovery group $(678.67 \pm 810 \mu \mathrm{L})$.

Table 1 - Test results before (Pre) and after (Post) two motorcycle races separated by a passive recovery protocol. Data are shown as mean (standard deviation).

\begin{tabular}{|c|c|c|c|c|c|c|c|c|}
\hline & Handgrip Strength (N) & Blood Lactate $(\mathrm{mmol} / \mathrm{L})$ & HR (bpm) & UBP (N) & Ball Throw (m) & Lap times (s) & CMJ (m) & RT (s) \\
\hline \multirow[t]{2}{*}{ Pre race 1} & 77.5 & 1.17 & & 140.1 & 5.6 & & 0.27 & 0.73 \\
\hline & (7.7) & $(0.34)$ & & $(16.6)$ & $(0.8)$ & & $(0.04)$ & $(0.10)$ \\
\hline \multirow[t]{2}{*}{ Post race 1} & $59.4^{\mathrm{a}}$ & $3.89^{\mathrm{a}}$ & 175 & 121.5 & 5.51 & 130.5 & 0.27 & 0.72 \\
\hline & (11.1) & $(1.31)$ & (6) & $(16.5)$ & $(0.71)$ & (9.6) & $(0.04)$ & $(0.06)$ \\
\hline \multirow[t]{2}{*}{ Pre race 2} & $67.3^{\mathrm{a}}$ & $1.71^{\mathrm{b}}$ & & 134.7 & 5.55 & & 0.26 & 0.67 \\
\hline & $(10.2)$ & $(0.64)$ & & $(20.6)$ & $(0.66)$ & & $(0.05)$ & $(0.09)$ \\
\hline \multirow[t]{2}{*}{ Post race 2} & $61.2^{\mathrm{a}}$ & 2.80 & 172 & 122.5 & 5.43 & 130.5 & 0.28 & $0.66^{\mathrm{a}, \mathrm{b}}$ \\
\hline & $(10.3)$ & $(1.48)$ & (11) & $(25.4)$ & $(0.54)$ & $(10.5)$ & $(0.05)$ & $(0.10)$ \\
\hline \multirow[t]{2}{*}{ Post $24 \mathrm{~h}$} & $75.2^{\mathrm{d}}$ & & & 144.6 & & & & \\
\hline & $(10.2)$ & & & (31.3) & & & & \\
\hline
\end{tabular}

CMJ, counter movement jump; UBP, upper body pull; HR, heart rate; RT, Reaction time; CK, creatine kinase. a = statistically significant difference compared to Pre race $1 ; \mathrm{b}=$ statistically significant difference compared to Post race $1 ; \mathrm{c}=$ statistically significant difference compared to Pre race $2 ; \mathrm{d}=$ statistically significant difference compared to Pre race 2 . 
Table 2 - Test results before (Pre) and after (Post) two motorcycle races separated by an active recovery protocol. Data are shown as mean (standard deviation).

\begin{tabular}{|c|c|c|c|c|c|c|c|c|}
\hline & Handgrip Strength (N) & Blood Lactate $(\mathrm{mmol} / \mathrm{L})$ & HR (bpm) & UBP (N) & Ball Throw (m) & Lap times (s) & $\mathbf{C M J}(\mathbf{m})$ & RT (s) \\
\hline \multirow[t]{2}{*}{ Pre race 1} & 82.4 & 1.26 & & 135.2 & 5.61 & & 0.25 & 0.73 \\
\hline & $(16.3)$ & $(0.36)$ & & $(16.2)$ & $(0.59)$ & & $(0.04)$ & $(0.11)$ \\
\hline \multirow[t]{2}{*}{ Post race 1} & $59.8 \mathrm{a}$ & $5.58^{\mathrm{a}}$ & 179 & 127.1 & 5.7 & 127.8 & 0.28 & 0.70 \\
\hline & $(11.8)$ & $(3.21)$ & (9) & $(23.5)$ & $(0.75)$ & $(10.9)$ & $(0.03)$ & $(0.08)$ \\
\hline \multirow[t]{2}{*}{ Pre race 2} & $68.3 \mathrm{a}$ & $1.85^{\mathrm{b}}$ & & 130.0 & 5.59 & & 0.26 & 0.67 \\
\hline & $(15.1)$ & $(0.86)$ & & $(24.6)$ & $(0.82)$ & & $(0.05)$ & $(0.09)$ \\
\hline \multirow[t]{2}{*}{ Post race 2} & $64.9^{\mathrm{a}}$ & $4.76^{\mathrm{a}, \mathrm{c}}$ & 181 & 117.5 & 5.73 & 128.2 & 0.29 & $0.61^{\mathrm{a}, \mathrm{b}}$ \\
\hline & $(20.8)$ & $(2.62)$ & $(5)$ & $(30.7)$ & $(0.71)$ & $(11.1)$ & $(0.04)$ & $(0.08)$ \\
\hline \multirow[t]{2}{*}{ Post $24 \mathrm{~h}$} & 75.6 & & & $147.2^{\mathrm{b}}$ & & & & \\
\hline & $(13.7)$ & & & $(23.7)$ & & & & \\
\hline
\end{tabular}

CMJ, counter movement jump; UBP, upper body pull; HR, heart rate; RT, Reaction time; CK, creatine kinase. a = statistically significant difference compared to Pre race $1 ; \mathrm{b}=$ statistically significant difference compared to Post race $1 ; \mathrm{c}=$ statistically significant difference compared to Pre race $2 ; \mathrm{d}=$ statistically significant difference compared to Pre race 2 .

\section{Discussion}

This study showed that active recovery in between motocross races does not have a positive effect on specific motocross performance, strength, power or reaction time. Furthermore, active or passive recovery protocols had no effect on markers of muscle damage one-day following the motocross racing. In both recovery protocols there were increases in blood lactate and decreases in isometric strength, only during race 1 . These results are in line with the previous studies that has investigated motocross racing $^{4,3,18,19}$.

Against our hypothesis, active recovery was not superior to passive recovery when comparing performance values. Strength and power tests did not improve when active recovery was performed by the subjects. Motocross racing is a predominantly aerobic exercise ${ }^{18}$, and it seems that active recovery might be beneficial when anaerobic contribution is more considerable ${ }^{13,14}$. In addition, active recovery seems to be superior when short time periods are placed in between high intensity exercise ${ }^{13,14,34}$. In this study, the break in between the simulated races was $1 \mathrm{~h}$, similar to what is normal during a race weekend. Likewise, active recovery could harm muscle glycogen resynthesis and thus subsequent performance when implemented in between exercise bouts ${ }^{12}$. Maybe with a recovery protocol involving also the musculature of the arms and upper body, the results could have been different as some recent evidence suggests ${ }^{23}$.

Reaction time is important in motorsports, as it can be essential for success and is a key factor in order to avoid injury while riding ${ }^{7,9}$. RT scores did not vary between active and passive recovery protocols, even

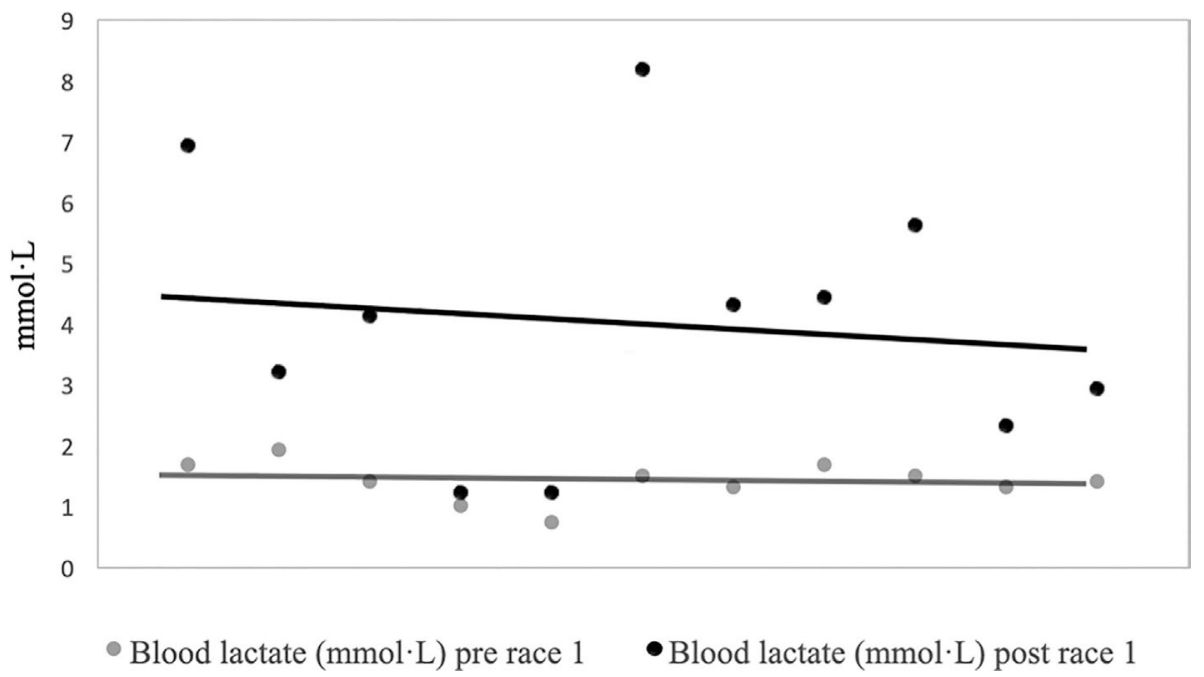

Figure 3 - A significant increase was found when comparing Pre and Post race 1 blood lactate values, before the recovery protocol was performed. 
though, the scores improved every time the participants performed the test, suggesting maybe some type of learning effect.

This is the first study that assessed indirect markers of muscle damage after motocross racing. CK values obtained $24 \mathrm{~h}$ Post racing were high compared to what is considered resting values ${ }^{17}$. With the results obtained, it seems that after motocross racing muscle damage may exist in elite and non-elite racers. However, the active recovery in between motocross races did not have a significant effect on the present muscle damage markers. The present results go in hand with the data presented by Andersson et al. ${ }^{2}$ and Wigernaes et al. ${ }^{38}$ in which the participants that performed active recovery after the exercise bouts did not show lower CK values. The hypothesis of the study has been refuted, as the use of active recovery does not seem to accelerate muscle damage recovery, but importantly, it does not harm recovery as well.

\section{Conclusion}

In conclusion, when compared to passive recovery, active recovery on a cycle ergometer does not seem to enhance motocross riding performance, strength and power production. Also, active recovery does not improve reaction time of motocross riders. It is possible that some degree of muscle damage after a simulated motocross race exists, but the muscle damage it is not affected by the use of active or passive recovery in between races. Coaches and athletes can use the present kind of active recovery in between races if subjective perception of recovery is higher, as performance will not be harmed. However, it should be kept in mind that muscle damage could exist after racing. Therefore, a recovery period over one day could be necessary after a race weekend in order to reach baseline levels in all physiological parameters. Maximal isometric handgrip strength could be an easy and quick tool to assess fitness state of the motocross riders after a race. Future research should test high-level riders during a competition. Even tough participants were encouraged to give their best at all times, the intensity of a practice day can never match a real race.

\section{Acknowledgments}

We acknowledge the Keski Suomi Moottorikerho for providing the facilities for the study. This research was supported financially by the Finnish Motorcycling Federation (Suomen Moottoriliitto ry).

\section{References}

1 Ascensão A, Ferreira R, Marques F, Oliveira E, Azevedo V, Soares J, et al. Effect of off-road competitive motocross race on plasma oxidative stress and damage markers. Brit $\mathrm{J}$ Sports Med. 2007;41(2):101-5.
2 Konttinen T, Häkkinen K, Kyröläinen H. Cardiopulmonary loading in motocross riding. J Sports Sci. 2007;25(9):9959.

3 Ascensão A, Azevedo V, Ferreira R, Oliveira E, Marques F, Magalhães J. Physiological, biochemical, and functional changes induced by a simulated 30 min off-road competitive motocross heat. J sports Medicine Phys Fitness. 2008;48 (3):311-9.

4 Konttinen T, Kyröläinen H, Häkkinen K. (2008). Cardiorespiratory and neuromuscular responses to motocross riding. $\mathrm{J}$ Strength Cond Res. 2008;22(1):202-9.

5 Beneke R, Leithauser RM, Ochentel O. Blood lactate diagnostics in exercise testing and training. Int $\mathrm{J}$ Sports Physiol Perform. 2011;6(1):8-24.

6 French DN, Kraemer WJ, Volek JS, Spiering BA, Judelson DA, Hoffman JR, et al. Anticipatory responses of catecholamines on muscle force production. J Appl Physiol. 2007; 102 (1):94-102.

7 Kreisman SH, Ah Mew N, Halter JB, Vranic M, Marliss EB. (2001). Norepinephrine Infusion during Moderate-Intensity Exercise Increases Glucose Production and Uptake 1. J Clin Endocrinol \& Metabol. 2001;86(5):2118-24.

8 Zouhal H, Jacob C, Delamarche P, Gratas-Delamarche A. Catecholamines and the effects of exercise, training, and gender. Sports Med. 2008;38(5):401-23.

9 Rusch NJ, Shepherd JT, Webb RC, Vanhoutte PM. Different behavior of the resistance vessels of the human calf and forearm during contralateral isometric exercise, mental stress, and abnormal respiratory movements. Circ Res. 1981;48(6 Pt 2):I118-30.

10 Smolander J, Aminoff T, Korhonen I, Tervo M. Shen N, Korhonen O, Louhevaara V. Heart rate and blood pressure responses to isometric exercise in young and older men. Eur J Appl Physiol Occup Physiol. 1998;77(5):439-44.

11 Devlin J, Paton B, Poole L, Sun W, Ferguson C, Wilson, J, et al. Blood lactate clearance after maximal exercise depends on active recovery intensity. J Sports Med Phys Fitness. 2014;54(3):271-8.

12 Barnett A. Using recovery modalities between training sessions in elite athletes. Sports Med, 2006;36(9):781-96.

13 Ali RS, Koushkie JM, Asadmanesh A, Salesi M. Influence of massage, active and passive recovery on swimming performance and blood lactate. J Sports Med Phys Fitness. 2012;52 (2):122-7.

14 Dorado C, Sanchis-Moysi J, Calbet JA. Effects of recovery mode on performance, $\mathrm{O} 2$ uptake, and $\mathrm{O} 2$ deficit during high-intensity intermittent exercise. Can J Appl Physiol. 2004;29(3):227-44.

15 White GE, Wells GD. The effect of on-hill active recovery performed between runs on blood lactate concentration and fatigue in alpine ski racers. J Strength Cond Res. 2015;29 (3):800-6.

16 Sahlin K, Harris RC, Hultman E. Resynthesis of creatine phosphate in human muscle after exercise in relation to intramuscular $\mathrm{pH}$ and availability of oxygen. Scand J Clin Lab Invest. 1979;39(6):551-7.

17 Garner E. The Effects of Active and Passive Recovery on Performance in Women's Collegiate Basketball Athletes. Int J Exerc Sci: Conference Proc. 2016;(2):38. 
18 Losnegard T, Andersen M, Spencer M, Hallén J. Effects of Active Versus Passive Recovery in Sprint Cross-Country Skiing. Int J Sports Physiol Perform. 2015;10(5):630-5.

19 Rey E, Lago-Peñas C, Lago-Ballesteros J, Casáis, L. The effect of recovery strategies on contractile properties using tensiomyography and perceived muscle soreness in professional soccer players. J Strength Cond Res. 2012;26 (11):3081-88.

20 Taipale RS, Heinaru S, Nindl BC, Vaara JP, Santtila M. Häkkinen K. et al. Hormonal Responses to Active and Passive Recovery After Load Carriage. J Strength Cond Res. 2015;29:S149-S153.

21 Tessitore A, Perroni F, Cortis C, Meeusen R, Lupo C, Capranica L. Effects of different recovery interventionson anaerobic performances following preseason soccer training players. J Strength Cond Res. 2007;21(3):745-50.

22 Andersson HM, Raastad T, Nilsson J, Paulsen G, Garthe I, Kadi F. Neuromuscular fatigue and recovery in elite female soccer: effects of active recovery. Med Sci Sports Exerc. 2008;40(2):372-80.

23 Scanlan AT, Madueno MC. Passive recovery promotes superior performance and reduced physiological stress across different phases of short-distance repeated sprints. J Strength Cond Res. 2016;30:2540-9.

24 Wahl P, Mathes S, Köhler K, Achtzehn S, Bloch W, Mester J. Effects of active vs. passive recovery during Wingatebased training on the acute hormonal, metabolic and psychological response. Growth Horm IGF Res. 2013;23(6):201-8.

25 Wahl P, Zinner C, Grosskopf C, Rossmann R, Bloch W, Mester J. Passive recovery is superior to active recovery during a high-intensity shock microcycle. J Strength Cond Res. 2013;27(5):1384-93.

26 Fairchild TJ, Armstrong AA, Rao A, Liu H, Lawrence S, Fournier PA. Glycogen synthesis in muscle fibers during active recovery from intense exercise. Med Sci Sports Exerc. 2003;35(4):595-602.

27 Newham DJ, McPhail G, Mills KR, Edwards RHT. Ultrastructural changes after concentric and eccentric contractions of human muscle. J Neurol Sci. 1983;61(1):109-22.

28 Avela J, Kyröläinen H, Komi PV, Rama D. Reduced reflex sensitivity persists several days after long-lasting stretchshortening cycle exercise. J Appl Physiol. 1999;86(4):1292300.

29 Tanaka H, Monahan KD, Seals DR. Age-predicted maximal heart rate revisited. J Am Coll Cardiol. 2001;37(1):153-6.

30 Maughan RJ, Leiper J, Shirreffs SM. Factors influencing the restoration of fluid and electrolyte balance after exercise in the heat. Brit J Sports Med. 1997;31(3):175-82.
31 Osterberg KL, Pallardy SE, Johnson RJ, Horswill CA. Carbohydrate exerts a mild influence on fluid retention following exercise-induced dehydration. J Appl Physiol. 2009;108 (2):245-50.

32 Franchini E, Bertuzzi, RCM, Takito, MY, Kiss, MAPDM. Effects of recovery type after a judo match on blood lactate and performance in specific and non-specific judo tasks. Eur J Appl Physiol. 2009;107(4):377-83.

33 Franchini E, Takito MY, Nakamura FY, Matsushigue KA, Kiss MPDM. Effects of recovery type after a judo combat on blood lactate removal and on performance in an intermittent anaerobic task. J Sports Med Phys fitness. 2003;43(4):42431.

34 Van Hooren B, Peake JM. Do We Need a Cool-Down After Exercise? A Narrative Review of the Psychophysiological Effects and the Effects on Performance, injuries, and the long-term adaptive response. Sports Med. 2018;48:1575-95.

35 Baur H, Müller S, Hirschmüller A, Huber G, Mayer F. Reactivity, stability, and strength performance capacity in motor sports. Brit J Sports Med. 2006;40(11):906-11.

36 Mika A, Oleksy Ł, Kielnar R, Wodka-Natkaniec E, Twardowska M, Kamiaski K, et al. Comparison of two different modes of active recovery on muscles performance after fatiguing exercise in mountain canoeist and football players. PloS one. 2016;11(10):e0164216.

37 Baur H, Müller S, Hirschmüller A, Huber G, Mayer F. Reactivity, stability, and strength performance capacity in motor sports. Brit J sports Med. 2006;40(11):906-11.

38 Daniels DJ, Clarke MJ, Puffer R, Luo TD, McIntosh AL, et al. High occurrence of head and spine injuries in the pediatric population following motocross accidents. J Neurosurgery: Pediatrics. 2015;15(3):261-5.

\section{Corresponding author}

Aleix Ollé Casanovas. 17 Russell Court, OX5 1GJ, Kidlington, United Kingdom.

E-mail: aleix.olle.casanovas@hintsa.com.

Manuscript received on January 30, 2021

Manuscript accepted on April 29, 2021

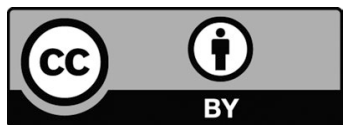

Motriz. The Journal of Physical Education. UNESP. Rio Claro, SP, Brazil - eISSN: 1980-6574 - under a license Creative Commons - Version 4.0 